\title{
Phenotyping Asthma: Linking Clinical Features to Biomolecular Pathways
}

Kian Fan Chung MD DSc FRCP FERS

Experimental Studies, National Heart \& Lung Institute, Imperial College London \& NIHR Biomedical Research Unit, Royal Brompton Hospital, London, UK

\section{ABSTRACT}

Asthma presents in various clinical forms and levels of severity and has a complex pathophysiology. Unbiased clustering was initially performed on clinical features, but the addition of biomarkers such as sputum and blood cellular profiles has led to the description of several phenotypes and enabled the prediction of responses to targeted therapies. Clusters of severe asthma include those on high-dose corticosteroid treatment, often with both inhaled and oral treatment, associated with severe airflow obstruction. Concordance between symptoms and sputum eosinophilia is observed in an eosinophilic inflammation-predominant group with few symptoms and late-onset disease who have a high prevalence of rhinosinusitis, aspirin sensitivity, and exacerbations. Sputum or blood eosinophilia is also a biomarker that can predict therapeutic responses to antibody-based treatments to block the effects of the T-helper- 2 cytokine, interleukin-5. Low T-helper-2 expression predicts poor therapeutic response to inhaled corticosteroid therapy. Much less is known about 'non-eosinophilic' or non-T-helper-2 asthma. Clustering on transcriptomic and/or proteomic data is leading to definition of molecular phenotypes and potential mechanisms. The definition of endotypes and biomarkers of disease and therapeutic responses will pave the way towards personalized medicine and healthcare for asthma. For the clinician, these will translate into useful tools and means for managing patients with asthma, particularly severe asthma. (BRN Rev. 2016;2:82-97) Corresponding author: Kian Fan Chung, f.chung@imperial.ac.uk

Key words: Asthma. Bioinformatics. Hierarchical clustering. Phenotyping.

\section{Correspondence to:}




\section{THE COMPLEXITY OF ASTHMA AND PHENOTYPING APPROACHES}

Asthma is a disease with complex aetiologies and presentations with different natural histories and outcomes. Initially defined simply as a condition that causes symptoms such as wheezing, shortness of breath, chest tightness, and cough that vary over time in their occurrence, frequency, and intensity and associated with variable expiratory airflow, there has been the later recognition that there is an important inflammatory process occurring in the airways that underlies the pathophysiology. Treatment approaches for asthma have been based on this notion, such that anti-inflammatory treatments based mainly on inhaled corticosteroid therapies with supportive bronchodilator therapies using $\beta$-adrenergic agonists have become the uniformly applied treatments ${ }^{1}$. Guidelines for asthma management, first widely disseminated in the 1990s, have focused on a uniform stepwise escalation of treatments (inhaled corticosteroids and $\beta$-adrenergic bronchodilators) irrespective of the phenotype or aetiology, with the level of treatment given commensurate with the severity of asthma in a particular patient (Global Initiative for Asthma, GINA). However, this approach to asthma management, while successful in a large proportion of patients with asthma, was not effective in controlling asthma in a significant proportion of asthma patients. This group encompassed patients labelled as "difficult-to-treat asthma", with a large proportion being truly refractory or insensitive to the effects of asthma therapies, including corticosteroids and $\beta$-adrenergic agonists, patients that are now grouped under the umbrella term of "severe asthma"2.
In trying to understand the pathophysiology of severe asthma, it was quite clear that this group of patients was heterogeneous in terms of their presentation, comorbid factors, type of underlying inflammation, and degrees of response to asthma treatments. This heterogeneity of asthma is now recognised within the definition of asthma of the recent GINA guidelines (http://www.ginasthma.org/documents/4). Indeed, in severe asthma, phenotype-guided treatment has been recognised with recommendation for the use of sputum-guided treatment to reduce exacerbations and/or steroid dose, and with the recent use of targeted add-on anti-immunoglobulin E (IgE) treatment for severe allergic asthma and consideration of addition of leukotriene receptor antagonists for treating aspirin-exacerbated asthma. Despite these advances in the management of asthma, the science of phenotyping asthma itself has remained fairly rudimentary until recently. Clinicians and epidemiologists have been aware of the varied presentation of asthma, and epidemiologists of the different outcomes of asthma, particularly in childhood, and of the differences in responsiveness to currently available treatments.

With the application of cluster analysis, an unbiased statistical technique for grouping sets of data that have a degree of closeness, there has been real progress made in defining clinical phenotypes based on clinical-physiological characteristics. Clustering requires approaches that will group a set of objects depending on the degree of closeness to each other so that objects in the same group are more similar to each other than those in other groups (or clusters). It is used in exploratory data mining, and is a widely used 
technique for statistical data analysis used in many fields including the determination of phenotypes of asthma dependent on disease characteristics. Of the many clustering algorithms in use, one commonly applied to asthma phenotyping has been connectivity models such as hierarchical clustering based on distance connectivity or $\kappa$-means algorithm base by single mean cluster.

While the definition of phenotypes on the basis of clinical parameters is useful to the clinician, it does not allow for an understanding of the mechanism underlying the phenotypic class, which is necessary for providing a more rational approach to management. Inclusion of risk factors, triggers, and biomarkers of inflammation in the clustering has led to an improvement in understanding of the complex interactions of many risk factors and underlying inflammatory pathways involved in asthma. The availability of -omics data has opened up another dimension to asthma phenotyping as inclusion of such data in the analysis opens up the possibility of defining groups of asthma depending on the pathophysiological/molecular mechanisms leading to the definition of molecular phenotypes of asthma. Finally, when these mechanisms have been shown to be underlying the clinical-physiological inflammatory processes of that phenotype, we can then define endotypes of asthma ${ }^{3}$. Endotypes will be most useful in defining targets for the development of new therapies and treatments for different endotypes of asthma, an important path towards the personalisation of treatment approaches ${ }^{4}$. Integration of biological and -omics data with clinical-physiological inflammatory parameters is challenging and will require mathematical modelling such as machine learning approaches or network models; in fact, new methods for integrative data analyses are required to overcome the computational challenges of these methods ${ }^{5}$ (Fig. 1).

This review will focus on the approaches to phenotyping asthma over the last 90 years, summarise the phenotypes of asthma that have been described so far, and point to the future directions of asthma phenotyping.

\section{PHENOTYPES BASED ON CLINICAL- PHYSIOLOGICAL PARAMETERS}

Clinicians have long recognised that asthma is a syndrome consisting of different phenotypes. Therefore, there have been clinical descriptors of asthma that can be loosely termed as "phenotype" based on essentially clinical-physiological parameters and biomarkers, a list of which is provided in table 1. One of the first clinical subgroups of asthma proposed has been based on the presence of allergic sensitisation with asthma, termed extrinsic asthma, as opposed to intrinsic asthma which is not associated with atopic disease, a division based on the notion that extrinsic asthma results from the exposure to common aeroallergens ${ }^{6}$. Extrinsic asthma was usually associated with a younger age of onset, while intrinsic asthma was usually associated with a later onset of asthma and a generally worse prognosis. Amongst the intrinsic group, late-onset asthma was a group of asthma with aspirin sensitivity and nasal polyposis that was usually associated with eosinophilia?. A recent systematic review of the literature regarding age of onset found that adults with early onset current asthma were more likely to be atopic and have more frequent symptoms 


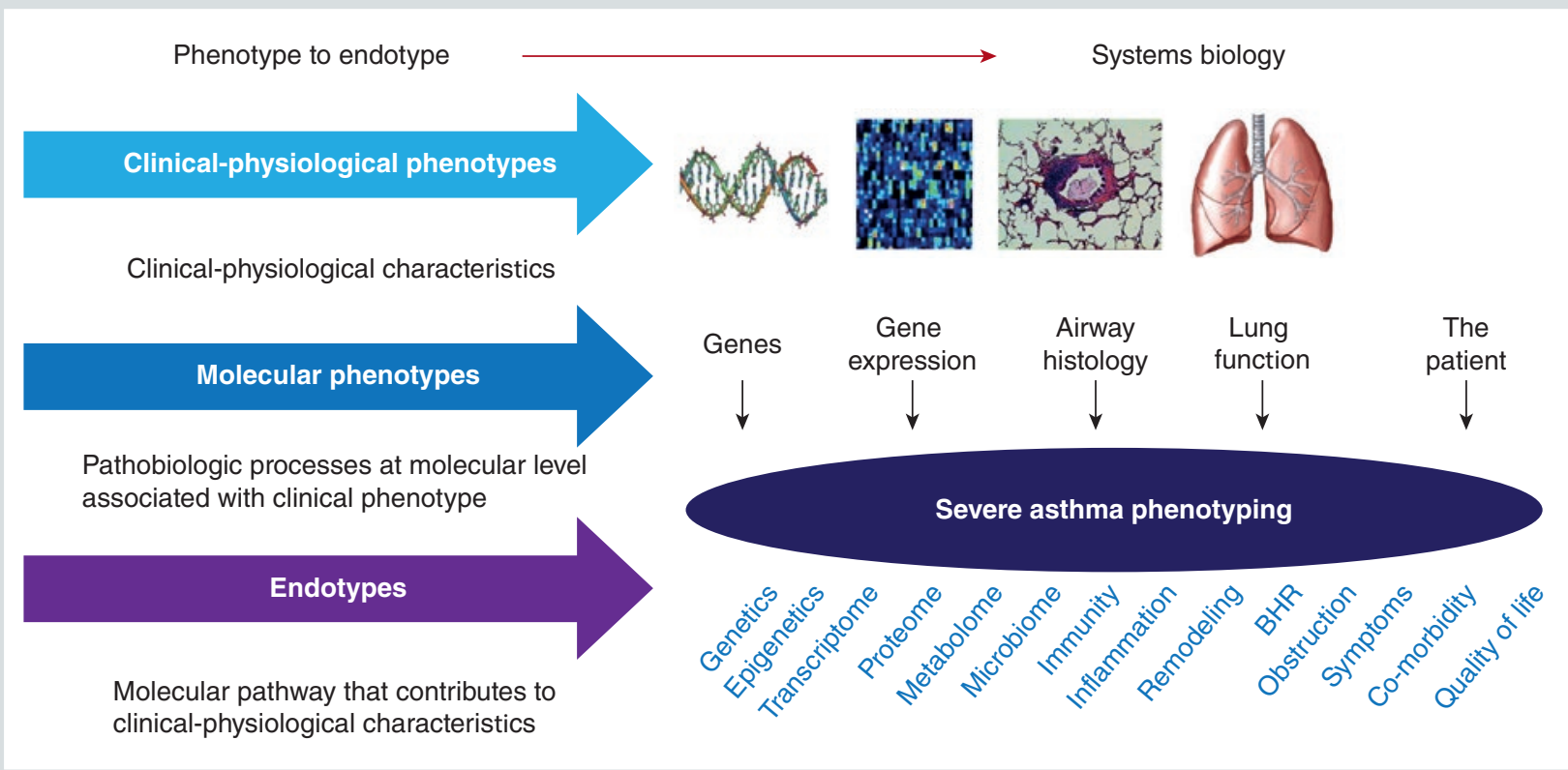

Figure 1. The definition of clinical-physiological and molecular phenotypes, ending up with endotypes where molecular pathways contribute to clinical-physiological characteristics. The process of phenotyping to endotyping relies on the science and mathematics of systems biology that links clinical features, physiology, pathology, gene and protein expression, metabolomics, and microbiome. BHR: bronchial hyperresponsiveness.

compared with those with late-onset disease, who were more likely to be female, smokers, and with a greater level of fixed airflow obstruction. However, in both groups the prevalence of severe asthma was similar with few phenotypic differences between severe asthmatics regardless of age of onset ${ }^{8}$. Whether the entity of intrinsic asthma versus extrinsic asthma is valid anymore continues to be debated as the clinical and immunopathological features of these two conditions appear to be similar 9 . Another category of patients recognised in the late 1950s was that of therapeutic responders to oral corticosteroid therapies versus those who were non-responders (corticosteroid-insensitive) and with the observation that blood or sputum eosinophilia was a marker of responsiveness to oral corticosteroid treatment, one of the first biomarkers of therapeutic response to be described for asthma ${ }^{10,11}$.

Other phenotypes have been recognised, which can be classified on the broad category according to causation, patterns of airflow obstruction, disease severity, and type of airway inflammation ${ }^{12}$ (Table 1). Asthma phenotypes according to patterns of airflow obstruction measured by peak expiratory flow rates or forced expiratory volume in one second $\left(\mathrm{FEV}_{1}\right)$ have been most studied. Fixed airflow limitation is recognised as a distinct phenotype of severe asthma found in up to $50 \%$ of patients who have a longer duration of disease, greater eosinophilic inflammation in sputum or blood, with thickening of the airways as seen on high resolution computed tomographic scans ${ }^{13,14}$. A greater 
TABle 1. Phenotypes of asthma

1. According to causal or trigger factor
Atopy
Aspirin
Infection
Occupation
Exercise
Obesity

2. According to type of airflow obstruction

Brittle asthma

Irreversible/fixed airflow obstruction

Yearly decline of lung function

3. According to severity and response to treatments

Mild-moderate asthma

Severe refractory asthma

Corticosteroid-insensitive asthma

Loss of $\beta$-agonist bronchodilator response

4. According to radiological findings

Airway dilatation

Bronchial wall thickening

Gas trapping

5. According to airway inflammation measured in sputum

Eosinophilic

Neutrophilic

Combined/mixed inflammatory

Paucigranulocytic

6. According to inflammatory biomarkers

Exhaled nitric oxide levels (FeNO)

Blood eosinophil count

Serum periostin

airway smooth muscle area associated with an increased expression of T-helper type 1 (Th1) and Th2 cytokines measured in sputum supernatants was seen in these patients ${ }^{15}$. Frequent exacerbators are also a well-defined phenotype because of its major source of morbidity and mortality, particularly in severe asthma. The risk factors associated with frequent exacerbators include severe sinus disease, gastro-oesophageal reflux disease, recurrent chest infections, psychological factors, and obstructive sleep apnoea ${ }^{16}$. These patients are characterised by lower quality of life scores, higher sputum eosinophilia, and a more rapid decline in $\mathrm{FEV}_{1}{ }_{17}$, and controlling the level of sputum eosinophilia with corticosteroid treatment led to a reduction in exacerbations in this phenotype, indicating the link between eosinophilia and exacerbations ${ }^{18}$. The role of triggers and precipitating factors in causing uncontrolled asthma and exacerbations may also be important in defining clinical clusters, but their direct effect remains unclear ${ }^{19,20}$.

\section{PHENOTYPING BASED ON CLUSTERING OF CLINICAL- PHYSIOLOGICAL DATA}

An unbiased approach to defining phenotypes using clustering techniques has been started by using cohorts of asthma with a range of clinical severities, initially using clinical-physiological correlates only, with the Severe Asthma Research Program (SARP) adult and paediatric cohorts ${ }^{21,22}$ and the United Kingdom (UK) Leicester adult cohorts ${ }^{23}$ being the pioneers in this rapidly expanding field. These studies reported phenotypes that have common, although not entirely similar, features that have already been recognized (Table 2), such as patients with little airflow obstruction and activity of disease, patients with early age of onset of disease with an atopic background, and a more severe group of asthma patients associated with adult-onset disease and active 
TABLE 2. Clusters of asthma

\begin{tabular}{|c|c|}
\hline \multicolumn{2}{|c|}{$\begin{array}{l}\text { A: Clusters of Severe Asthma Research Program (SARP) } \\
\text { adult cohort }{ }^{21}\end{array}$} \\
\hline Cluster 1 & $\begin{array}{l}\text { Early onset atopic asthma with normal lung } \\
\text { function treated with two or less controller } \\
\text { medications and minimal healthcare } \\
\text { utilization. }\end{array}$ \\
\hline Cluster 2 & $\begin{array}{l}\text { Early onset atopic asthma and preserved lung } \\
\text { function, but increased medication require- } \\
\text { ments and healthcare utilization. }\end{array}$ \\
\hline Cluster 3 & $\begin{array}{l}\text { Mostly older obese women with late-onset } \\
\text { non-atopic asthma, moderate reductions in } \\
\text { FEV }_{1} \text {, and frequent oral corticosteroid use to } \\
\text { manage exacerbations. }\end{array}$ \\
\hline $\begin{array}{l}\text { Clusters } \\
4 \text { and } 5\end{array}$ & $\begin{array}{l}\text { Severe airflow obstruction with bronchodilator } \\
\text { responsiveness, but differ in their ability to } \\
\text { attain normal lung function, age of asthma } \\
\text { onset, atopic status, and use of oral } \\
\text { corticosteroids. }\end{array}$ \\
\hline \multicolumn{2}{|c|}{ B: Clusters of secondary care Leicester cohort ${ }^{23}$} \\
\hline Cluster 1 & $\begin{array}{l}\text { Early onset atopic asthma, with airway } \\
\text { dysfunction and eosinophilic inflammation; } \\
\text { increased number of hospitalizations. }\end{array}$ \\
\hline Cluster 2 & $\begin{array}{l}\text { Non-eosinophilic inflammation. Obese, female } \\
\text { predominant. }\end{array}$ \\
\hline Cluster 3 & $\begin{array}{l}\text { Early onset, symptom predominant with } \\
\text { minimal eosinophilic disease. }\end{array}$ \\
\hline Cluster 4 & $\begin{array}{l}\text { Eosinophilic inflammation-predominant with } \\
\text { few symptoms, late-onset disease. }\end{array}$ \\
\hline
\end{tabular}

disease. Such clusters have been replicated in adult and paediatric cohorts in the Western world $^{24-27}$ as well as in Korean and Japanese cohorts $^{28,29}$, with greater concordance in clusters related to severe asthma. Patients on high-dose inhaled corticosteroid therapy made up of clusters 4 and 5 of the adult SARP cohort, often taken together with oral corticosteroid treatment, are usually associated with severe airflow obstruction (Fig. 2). In Korean patients with refractory asthma ${ }^{30}$, three of the four clusters described closely resembled clusters 4 and 5 of SARP, with the Korean cluster 4 consisting predominantly of male cigarette smokers. In The Epidemiology and
Natural History of Asthma: Outcomes and Treatment Regimens (TENOR) study, the fifth cluster, described in adolescents and adults, was associated with aspirin sensitivity, in primarily white, female, and atopic patients with late-onset asthma, and with increased exacerbation rates ${ }^{26}$. In the Childhood Asthma Management Program Research Group, spectral clustering applied to clinical data from 1,041 children with asthma led to the description of five reproducible clusters differentiated on the basis of atopic burden, degree of airway obstruction, and history of exacerbations. Cluster grouping predicted long-term asthma control assessed by need for controller medication including oral prednisolone, and by longitudinal differences in lung function ${ }^{27}$.

The EU-funded Unbiased Biomarkers for the Prediction of Respiratory Disease Outcomes (U-BIOPRED) project has just published details of their paediatric and adult severe asthma cohorts ${ }^{31,32}$. This cross-sectional assessment of adults with severe asthma, mild/ moderate asthma, and healthy controls from 11 European countries showed that patients with severe asthma had more symptoms and exacerbations (2.5 exacerbations versus 0.4 in the preceding 12 months) compared to patients with mild/moderate disease, with worse quality of life and higher levels of anxiety and depression. They also had a higher incidence of nasal polyps and gastro-oesophageal reflux with lower lung function. A preliminary report has shown four stable clusters derived from an analysis of eight clinical-physiological features, with one cluster consisting of well-controlled moderate-to-severe asthmatics, while the three other clusters of predominantly severe asthma consisted of (i) a group of late-onset severe asthmatics with history 


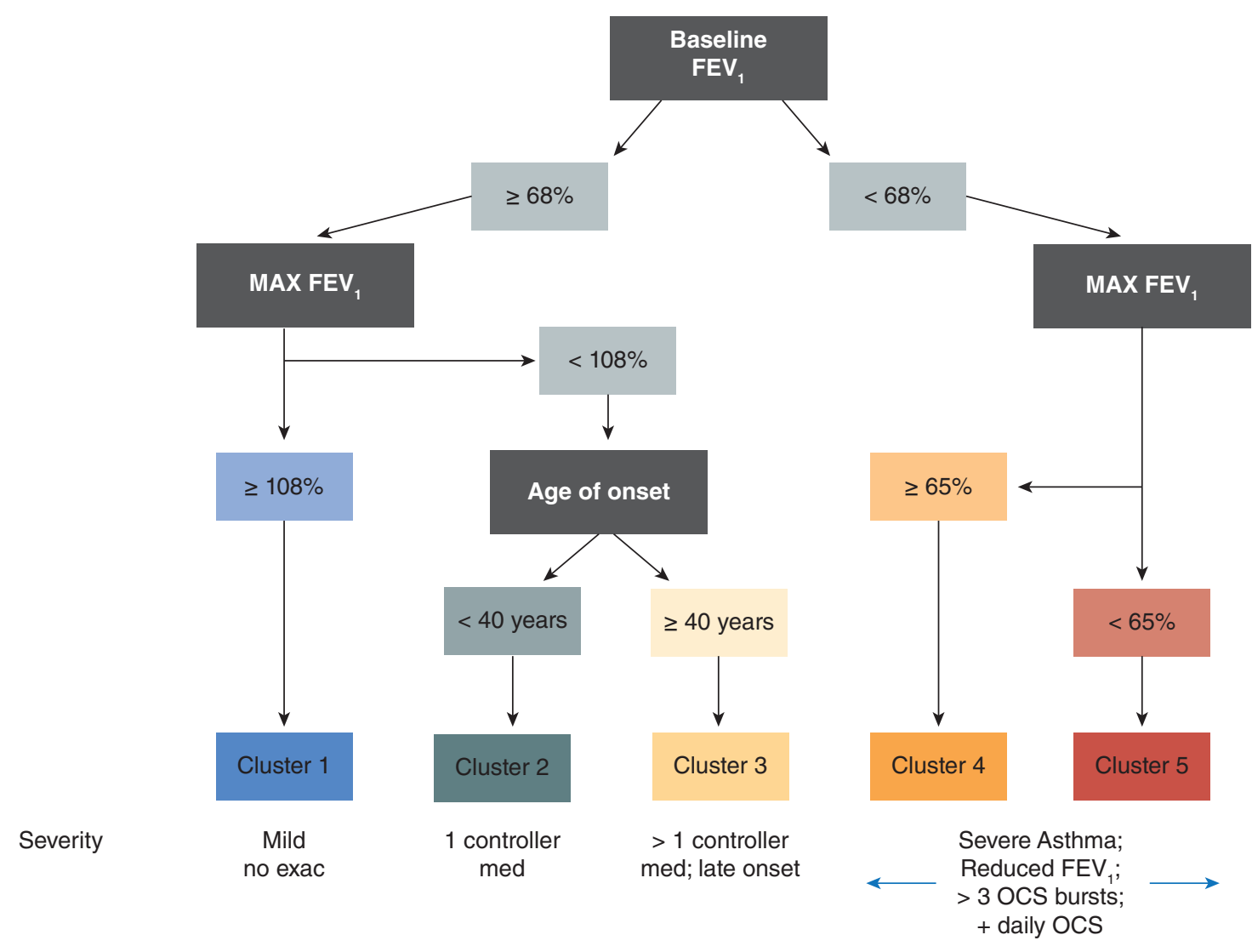

Figure 2. Clustering of 304 severe and 422 non-severe asthma patients in the Severe Asthma Research Program (SARP) cohort. This tree analysis shows that using only three physiological/historical variables (baseline FEV , maximal FEV $_{1}$ after 6-8 puffs of salbutamol, and age of onset of asthma), $80 \%$ of the asthma subjects could be assigned to five clusters from cluster 1 to cluster 5 . These clusters range from mildly severe (cluster 1 ) to the most severe (clusters 4 and 5) asthma patients (reproduced with permission from Moore et al. Am J Respir Crit Care Med. 2010;181:315-23).

Exac: exacerbation; $\mathrm{FEV}_{1}$ : forced expiratory volume in one second; MAX: maximal; med: medication; OCS: oral corticosteroids.

of smoking and chronic airflow obstruction, (ii) a group of chronic airflow obstruction but were non-smokers, and (iii) a group of obese female uncontrolled severe asthmatics with increased exacerbations, but with normal lung function $^{33}$. The sputum eosinophil counts were highest in the two obstructed clusters. These clusters exhibited similarities with the clusters reported in the SARP and the Leicester cohorts.

New clinical groups, such as those associated with obesity, have also been defined in both the SARP and Leicester cohorts. This has now been confirmed in other analyses that have specifically examined the contribution of obesity $^{34,35}$. Two clusters of obese individuals were described: obese uncontrolled and obese well-controlled, and these asthma clusters differed from one another with regard to age of asthma onset, measures of asthma symptoms and control, exhaled nitric oxide (FeNO) concentration, and airway hyper-responsiveness, but were similar with regard to measures of lung function, airway eosinophilia, 
and serum $\operatorname{Ig} \mathrm{E}^{34}$. A group of obese women with late-onset asthma and frequent symptoms with high healthcare use, but with low sputum eosinophil counts, has also been reported ${ }^{36}$. The finding of obesity associated clusters does not provide causation, but there is growing evidence that obesity itself may contribute to the inflammatory mechanisms of asthma ${ }^{37 .}$

\section{PHENOTYPING BASED ON SELECTED INFLAMMATORY BIOMARKERS}

The addition of inflammatory markers such as blood eosinophil counts or sputum eosinophils or levels of FeNO added a new dimension to the phenotyping. There has been confirmation that sputum eosinophils can be used as biomarkers of corticosteroid-sensitive asthma ${ }^{38,39}$, while non-eosinophilic asthma, defined by low sputum eosinophilia, is generally insensitive to the effects of corticosteroids. In fact, using sputum eosinophil counts to determine the amount of corticosteroid therapy needed to control asthma was superior to using symptoms in improving asthma control $^{18}$. Anti-inflammatory therapy with corticosteroids caused significant improvements in airflow obstruction in eosinophilic asthma, but not in persistently non-eosinophilic asthma. Non-eosinophilic asthma was more predominant in mild-to-moderate asthma, just as neutrophilic asthma is also predominant in severe refractory asthma ${ }^{40,41}$.

\section{Blood eosinophil counts}

Blood eosinophilia has now become an area of interest as a biomarker for elevated T2 cytokines. Interest has turned towards the use of blood eosinophilia as a biomarker of Th2 high and as a biomarker for responsiveness to anti-Th2 cytokines such as anti-interleukin 5 (anti-IL-5) or anti-IgE therapies for severe asthma. Blood eosinophilia is linked to elevated Th2 cytokines, but this relationship is not as strong as sputum eosinophilia ${ }^{42}$. On the other hand, in a group of uncontrolled treated asthmatic patients, blood eosinophil counts could accurately predict eosinophilic asthma ${ }^{43}$. Blood eosinophilia has also been linked to worse outcome measures in asth$\mathrm{ma}^{44,45}$, to more severe disease ${ }^{46}$, as a predictor of response to anti-IL-5 therapies ${ }^{47-50}$, and to anti-IgE therapy ${ }^{51,52}$ and to responsiveness to corticosteroid therapies ${ }^{53,54}$. Because it is easier to obtain blood eosinophil counts from patients than sputum eosinophil counts, blood eosinophil counts are now being used more widely in the clinic.

\section{Exhaled nitric oxide}

Similar to blood eosinophil counts, FeNO has been associated with worse asthma outcomes, the need for corticosteroid therapy, and responsiveness to corticosteroid therapy ${ }^{55}$. FeNO was predictive of response to anti $\operatorname{IgE}^{51}$. However, levels of FeNO have not been predictive of response to anti-IL-5 therapy ${ }^{56}$. On the other hand, therapies with anti-IL-4R $\alpha$ (interleukin-4 receptor $\alpha$ ) or anti-IL-13 antibodies caused a reduction in FeNO, but had no effect on blood eosinophil counts ${ }^{57-59}$.

\section{Serum periostin}

Periostin is a member of the Th2 genes induced by IL-13. Serum periostin has been shown to 
correlate with airway and sputum eosinophilia ${ }^{60}$ and is associated with Th2 inflammation $^{42}$. Serum periostin has also been shown in a Japanese asthma cohort on inhaled corticosteroid treatment to denote those with chronic airflow obstruction ${ }^{61}$. Serum periostin level was a predictor of response of $\mathrm{FEV}_{1}$ to anti-IL-13 therapy in patients with moderate-to-severe asthma ${ }^{57}$.

\section{PHENOTYPING BASED ON CLUSTERING OF CLINICAL- PHYSIOLOGICAL INFLAMMATORY BIOMARKERS}

In the Leicester cohorts where the sputum eosinophil count was a central parameter used, two clusters (the early onset atopic and the non-eosinophilic obese) were common to the milder-moderate and the moderate-severe cohorts $^{23}$. However, in the more severe cohort with refractory asthma, there was a marked discordance in two clusters between symptom expression and eosinophilic airway inflammation (early onset symptom predominant and late-onset inflammation predominant). However, in both discordant subgroups, an inflammation-guided management was superior in leading to a reduction in exacerbation frequency in the inflammation-predominant cluster and a dose reduction of inhaled corticosteroid in the symptom-predominant cluster. Thus, documentation of the sputum eosinophil level was useful in the therapeutic sense.

In the SARP cohort, use of the inflammatory phenotypes assessed by sputum cell counts led to the identification of four phenotypic clusters with two clusters of mild-to-moderate early onset allergic asthma with paucigranulocytic or eosinophilic sputum inflammatory cell patterns, and two other clusters with either neutrophilic or mixed inflammatory patterns with moderate-to-severe asthma with frequent healthcare use despite treatment with high doses of inhaled or oral corticosteroids and also reduced lung function ${ }^{62}$. A similar report from the cohort in Newcastle, Australia, described that patients with neutrophilic inflammation had a greater frequency of primary care doctor visits for asthma exacerbations and a high prevalence of chest infections in the previous 12 months, of rhinosinusitis and of gastro-oesophageal reflux symptoms when compared to eosinophilic asthma ${ }^{62}$.

Addition of other inflammatory biomarkers including FeNO, bronchoalveolar lavage (BAL) cell counts and levels of serum IgE was also examined in the SARP cohort, leading to the definition of six clusters that included one healthy control cluster and five other asthma clusters. In these five clusters that were similar to the previously reported five clusters of SARP obtained from clustering on only clinical-physiological parameters ${ }^{63}$, there was a late-onset eosinophilic asthma cluster associated with nasal polyposis and sinusitis ${ }^{64}$, a cluster also described in the Leicester cohort ${ }^{23}$.

\section{CLINICAL VALUE AND STABILITY OF PHENOTYPES}

Having defined clusters, is there any value of applying cluster analysis into the clinic? First, the stability of clusters need to be determined, an important issue given that asthma is a variable condition punctuated by exacerbation of the disease process. Adult asthma phenotypes identified by a clustering approach on two 
occasions, 10 years apart, in three separate cohorts of mild-moderate asthma were reported to be highly consistent, with the phenotypes mainly characterised by the level of asthma symptoms, allergic state, and pulmonary function ${ }^{65}$. This may not be surprising considering that these were mild-moderate asthmatics. In the BIOAIR (the study of longitudinal assessment of clinical course and BIOmarkers in Severe Chronic AIRway disease) cohort of more severe asthma, phenotypes determined by biomarkers such as blood eosinophil or neutrophil counts and FeNO were less stable than those defined by physiological variables such as $\mathrm{FEV}_{1}{ }^{66}$. The definition of asthma phenotypes may be improved by repeated measurements to account for the fluctuations in lung function in asthma, particularly severe asthma.

Can application of cluster analysis predict outcomes in individual patients? In a recent analysis where clusters were identified according to the SARP definition of five clusters in a cohort of severe asthma that has been followed-up for at least one year, none of the clusters predicted significant outcomes ${ }^{67}$. It has been suggested that the inclusion and integration of prospective data such as the effect of therapeutic interventions, environmental data regarding pollution for example, and triggers for clustering could enhance their value in the clinic.

\section{MOLECULAR PHENOTYPING USING MICROARRAY APPROACHES}

The Th2-high molecular phenotype was the first to be defined by Woodruff et al. ${ }^{68}$ who examined the gene signature of airway epithelial brushings from mild-moderate asthmatics according to the degree of expression of IL-13-inducible genes, periostin, chloride channel regulator 1 , and serpin peptidase inhibitor (Fig 3). The Th2-high asthmatic patients, constituting about $50 \%$ of the cohort, had a greater degree of bronchial hyper-responsiveness, higher serum IgE levels, greater blood and airway eosinophilia, sub-epithelial fibrosis, and airway mucin gene expression, and responded well to inhaled corticosteroid therapy $^{69}$. Those with a low Th2 signature showed little or no response to inhaled corticosteroid therapy. The frequency of the Th2-high molecular phenotype has been analysed by direct measurement of sputum cell gene expression of IL-4, IL-5, and IL-13, with 70\% of mild-to-moderate asthmatics defined as Th2-high, characterised by more severe measures of asthma and increased blood and sputum eosinophilia ${ }^{42}$.

Using whole genome gene expression microarrays, Baines et al. ${ }^{70}$ found that out of 277 differentially expressed genes between asthma inflammatory phenotypes, a sixgene signature that included Charcot Leyden crystal protein, carboxypeptidase $\mathrm{A} 3$, deoxyribonuclease 1-like 3, IL-1 $\beta$ and CXCR2 chemokine receptor could discriminate eosinophilic asthma from the other inflammatory types including neutrophilic asthma. Using gene expression profiling of induced sputum and unsupervised hierarchical clustering of these expression profiles led to the description of three phenotypes: (i) chronic airflow obstruction and less well-controlled asthma, increased FeNO, and sputum eosinophils; (ii) airflow obstruction and higher sputum neutrophils; and (iii) higher sputum macrophages and lower eosinophils and neutrophils, and 


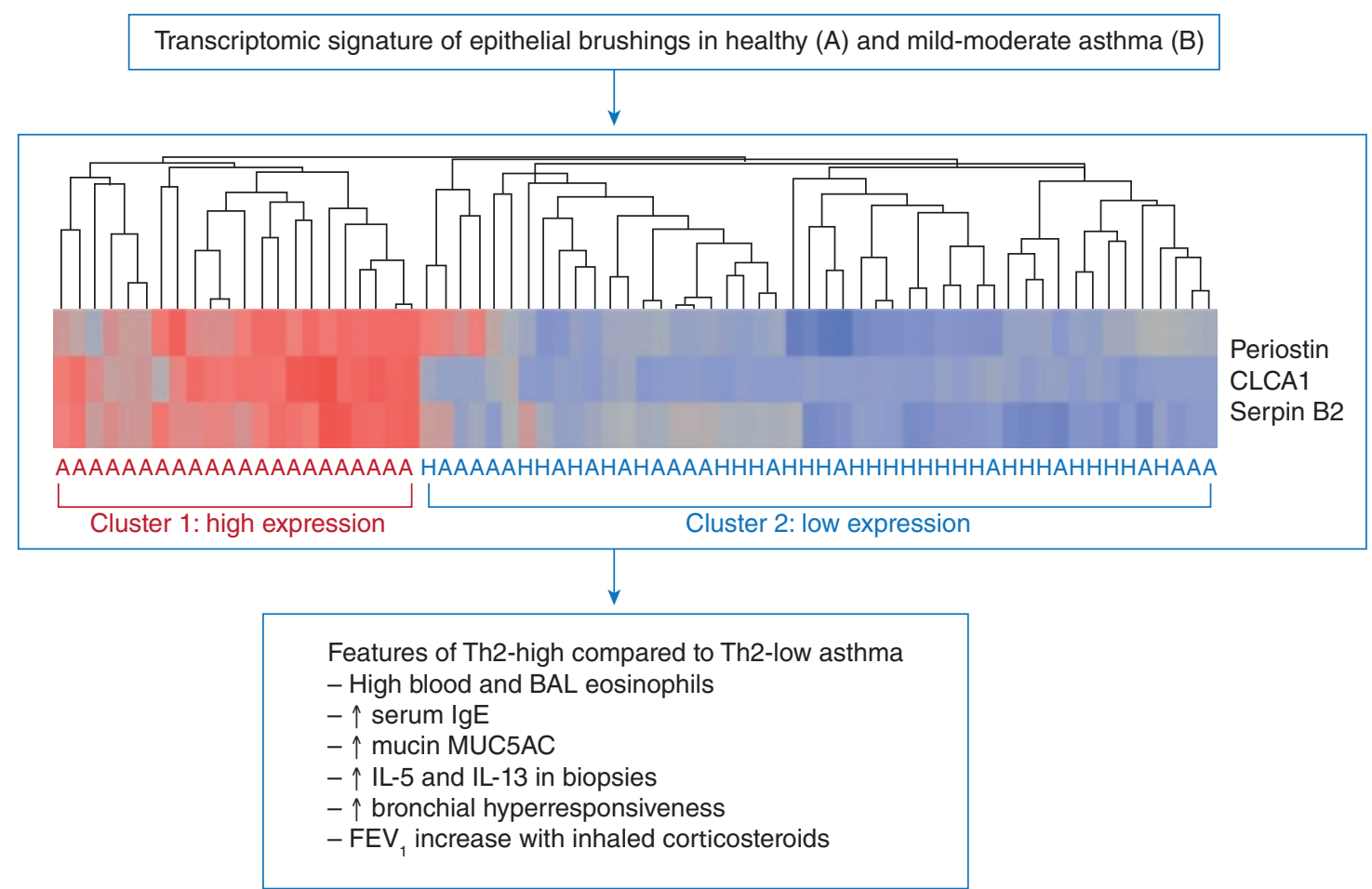

Figure 3. First transcription analysis defining the 'Th2-high' molecular phenotype of asthma. The expression of three genes that are up-regulated in airway epithelial cells exposed to the Th2 cytokine, IL-13 (periostin, CLCA1, serpin B2) in airway epithelial cells of healthy and mild-moderate asthma is shown. Nearly half of asthmatics show high Th2 expression (cluster 1 high expression) and the other half, a low Th2 expression (cluster 2 low expression). There are contrasting features between Th2-high and Th2-low subjects with higher eosinophil counts, bronchial hyperresponsiveness, and therapeutic response to corticosteroids in Th2-high patients (reproduced with permission from Woodruff et al. Amer J Respir Crit Care Med. 2009;180:388-95).

BAL: broncholaveolar lavage; CLCA1: calcium-activated chloride channel regulator 1 ; FEV ${ }_{1}$ : forced expiratory volume in one second; IgE: immunoglobulin E; IL: interleukin; Th: T-helper.

lung function in normal range. Genes in the IL-1 and tumour necrosis factor (TNF)- $\alpha /$ nuclear factor-кB pathways were also overexpressed and correlated with clinical parameters and neutrophilic airway inflammation.

Using a microarray platform to analyse bronchial airway epithelial cell gene expression in relation to the asthma biomarker fractional FeNO in SARP, five asthma clusters/phenotypes with distinct clinical, physiological, cellular, and gene transcription characteristics were described ${ }^{71}$. Genes related to type 2 inflammation were present, but other novel pathways, including those related to neuronal function, Wnt pathways, and actin cytoskeleton, were defined.

In UBIOPRED, using a semi-supervised machine-learning approach, a high mucosal eosinophils, high biopsy $\mathrm{CD}^{+}, \mathrm{CD}^{+}$, and $\mathrm{CD}^{+}$ T-cells, high FeNO and oral corticosteroid-dependent phenotype was derived from eight gene-signature sets applied to airway biopsy 
and brushings ${ }^{72}$. Another phenotype was characterized by high sputum eosinophilia, high biopsy $\mathrm{CD}^{+}$T-cells, and obesity. These phenotypes were not only dictated by a Th2high gene signature, but also by a Th1 signature, in addition to corticosteroid and oxidative response gene-sets.

Thus, these above approaches have led to the definition of novel non-Th2 pathways or genes in addition to the Th2 pathways/genes that may contribute to the molecular phenotyping of asthma. This is particularly true of pathways that are related to neutrophilic-type inflammation as defined in some studies. Bacterial colonization in the airways of patients with severe asthma could contribute to neutrophilic asthma ${ }^{73,74}$. Defective phagocytosis of bacteria or of apoptotic cells by macrophages has also been reported in severe asth$\mathrm{ma}^{75,76}$. More recent evidence, employing analysis of the microbiome using $16 \mathrm{~s}$, has shown differences in the bacterial organisation in the lower airways of patients with severe asthma by the presence of Streptococcus spp and Haemophilus influenzae associated with eosinophilia. Goleva et al. ${ }^{77}$ further showed that Haemophilus parainfluenzae overexpressed in severe asthma can directly induce corticosteroid resistance. Corticosteroids themselves can contribute to the neutrophilia to some extent, and even Th1 factors could play a role ${ }^{78,79}$.

Th17 cells have also been implicated as a cause of neutrophilia in severe asthma, perhaps even contributing to corticosteroid insensitivity ${ }^{80}$. In fact, there is evidence for an upregulation of the Th17 pathway in poorly controlled non-Th2 molecular phenotype ${ }^{80,81}$. Brodalumab, a human anti-IL-17R $\alpha$ monoclonal antibody, had no effect on asthma control scores, symptom-free days, and $\mathrm{FEV}_{1}$ in patients with inadequately controlled moderate-to-severe asthma on inhaled corticosteroid therapy. A high-reversibility subgroup with a post-bronchodilator $\mathrm{FEV}_{1}$ improvement of $20 \%$ or more showed a significant improvement in ACQ score ${ }^{82}$. The inflammasome could underlie neutrophilic asthma and has been shown to be activated in sputum cells from patients with severe neutrophilic asthma ${ }^{83,84}$.

\section{PHENOTYPE-DRIVEN THERAPIES}

With the recognition that the cytokines that were first identified to be produced from Th2 cells, such as IL-4, IL-5, and IL-13, can also be produced from other non-Th2 cell types, such as eosinophils, mast cells, and ILC-2, this inflammatory molecular phenotype has been labelled as Type-2 (T2) inflammation. Members of the Th2 pathway, such as IL-5, IL-4, and IL-13, have been targeted using humanised monoclonal antibodies directed towards these cytokines or their receptors and used as treatments for severe asthma ${ }^{85}$. Demonstration of efficacy of new therapies will depend in part on the precision by which patients can be endotyped for specific therapies ${ }^{4,86}$. Initially, the anti-IL-5 antibody mepolizumab was shown to have no beneficial effects on symptom control or exacerbations in a cohort of moderately severe asthmatics, despite reducing the levels of blood eosinophilia ${ }^{87}$. However, when the patients were recruited with having more severe asthma associated with a history of exacerbations and with persistent sputum or blood eosinophilia, mepolizumab decreased exacerbations, led to a reduction in the use of oral corticosteroids, and improved symptoms and lung function compared with placebo ${ }^{56,88}$. 
A blood eosinophil count of 150 cells/ $\mu$ l or more was associated with a reduction in exacerbation rate of $72 \%$ compared with a reduction of $30 \%$ in those with a blood eosinophil count of less than 150 cells/ $\mu$ l. Similar effects have also been demonstrated with another anti-IL-5 antibody, reslizumab, or an anti-IL-5 receptor antibody, benralizumab, in patients with blood eosinophilia ${ }^{50,89}$. However, we should still strive to find even better predictor biomarkers.

In patients with uncontrolled asthma despite use of inhaled corticosteroids and long-acting $\beta 2$ agonists, treatment with lebrikizumab, an anti-IL-13 antibody, improved $\mathrm{FEV}_{1}$ by $8.2 \%$ above the baseline value in patients with a high concentration of serum periostin, whereas patients with a low concentration of serum periostin showed no $\mathrm{FEV}_{1}$ response ${ }^{57}$.

These studies emphasise the importance of developing biomarkers for pinpointing those responders so that the right patient will get the right treatment, the real objective of personalised medicine ${ }^{90}$. What is currently missing is treatments focused on the non-Th2 pathways, although treatments based on targeting IL-17, CXC chemokines, and TNF- $\alpha$ have so far failed to show any benefits in severe asthma ${ }^{85}$.

\section{CONCLUDING REMARKS}

There have been important advances in the last 15 years in the definition of phenotypes, initially using clinical-physiological parameters and later with the inclusion of omics data, that has led to some definition of Th2 and non-Th2 pathways (Table 3). Much needs
TABLE 3. Disease drivers associated with asthma

1. Th2 pathway or T2 cytokines

2. Th1 pathway

3. Th17 pathway

4. Epithelial cell TSLP, IL-33 and IL-25

5. Interferon superfamily and response to infections

6. Inflammasome activation

7. Eosinophil activation

8. Neutrophil activation

9. Macrophage activation

10. Mast cell activation

11. B-cell activation

12. Oxidative stress pathways

13. Corticosteroid-inducible pathways

IL: interleukin; Th: T-helper; TSLP: thymic stromal lymphopoietin.

to be done to identify the non-Th2 pathways and their interactions with the Th2 pathway. At the time of writing, the data from UBIOPRED cohort have not been published yet, although there have been preliminary presentations at international meetings of some of the clustering findings based on an omics approach $^{72,84,91}$. What will emerge from these analyses is that there will likely be well-defined molecular phenotypes or endotypes underlined by multiple pathways that will include components of both the Th2 and nonTh2 pathways. The challenge will come in dissecting out the important pathway or gene components that are most important in a particular patient using the tools available for progressing systems medicine. The introduction of targeted therapies, particularly against Th2-associated cytokines such as IL-4, IL-5, and IL-13, has contributed to the validation of certain phenotypes such as the IL-5-dependent eosinophilic frequent exacerbator phenotype. Other non-Th2 components may be involved 
such as the inflammasome activation pathways, as has been reported recently ${ }^{92}$. It is important that any phenotype be validated in other independent cohorts, and therefore this area needs to represent a collaborative effort across major centres and also across national and continental boundaries to take into account the influence of local environmental and genetic factors.

Finally, it is important that the clinician is helped by knowledge of the phenotype of the asthma patient. This could be to provide longterm prediction of outcomes and to find out which specific treatments may benefit selected phenotypes. In effect, many would argue that phenotypes should confer predictive value and should relate to clinically meaningful outcomes. These aspects need to be addressed. Predictive value may be obtained if indicators of the day-to-day variability of the condition are included in the analysis. The -omics approach should contribute towards the availability of new, specific treatments while defining the appropriate patient groups for these specific treatments ${ }^{90}$. Biomarkers that are easily measurable and accessible in the clinic to help the clinician in classifying patients would represent an advance. Blood eosinophil counts are now useful in the clinic in defining the T2-phenotype, but other more precise biomarkers are needed for the definition of other phenotypes. Application of phenotyping to the clinic is the cornerstone of the progress towards personalised medicine ${ }^{4}$.

\section{REFERENCES}

1. Chung KF, Caramori G, Adcock IM. Inhaled corticosteroids as combination therapy with beta-adrenergic agonists in airways disease: present and future. Eur J Clin Pharmacol. 2009;65:853-71.
2. Chung KF, Wenzel SE, Brozek JL et al. International ERS/ATS guidelines on definition, evaluation and treatment of severe asthma. Eur Respir J. 2014;43:343-73.

3. Lotvall J, Akdis CA, Bacharier LB et al. Asthma endotypes: a new approach to classification of disease entities within the asthma syndrome. J Allergy Clin Immunol. 2011;127:355-60.

4. Chung KF. Defining phenotypes in asthma: a step towards personalized medicine. Drugs. 2014;74:719-28.

5. Gligorijevic V, Przulj N. Methods for biological data integration: perspectives and challenges. J R Soc Interface. 2015;12.

6. Rackemann FM. A working classification of asthma. Am J Med. 1947;3:601-6.

7. Samter M, Beers RF. Intolerance to aspirin. Clinical studies and consideration of its pathogenesis. Ann Intern Med. 1968;68:975-83.

8. Tan DJ, Walters EH, Perret JL et al. Age-of-asthma onset as a determinant of different asthma phenotypes in adults: a systematic review and meta-analysis of the literature. Expert Rev Respir Med. 2015;9:109-23.

9. Humbert M, Menz G, Ying $S$ et al. The immunopathology of extrinsic (atopic) and intrinsic (non-atopic) asthma: more similarities than differences. Immunol Today. 1999;20:528-33.

10. Brown HM. Treatment of chronic asthma with prednisolone; significance of eosinophils in the sputum. Lancet. 1958;2:1245-7.

11. Horn BR, Robin ED, Theodore J, Van Kessel A. Total eosinophil counts in the management of bronchial asthma. N Engl J Med. 1975;292:1152-5.

12. Nair P, Dasgupta A, Brightling CE, Chung KF. How to diagnose and phenotype asthma. Clin Chest Med. 2012;33:445-57.

13. Bumbacea D, Campbell D, Nguyen L et al. Parameters associated with persistent airflow obstruction in chronic severe asthma. Eur Respir J 2004;24:122-8.

14. ten Brinke A, van Dissel JT, Sterk PJ, Zwinderman AH, Rabe KF, Bel EH. Persistent airflow limitation in adult-onset nonatopic asthma is associated with serologic evidence of Chlamydia pneumoniae infection. J Allergy Clin Immunol. 2001;107:449-54.

15. Kaminska M, Foley S, Maghni K et al. Airway remodeling in subjects with severe asthma with or without chronic persistent airflow obstruction. J Allergy Clin Immunol. 2009;124:45-51.e1-4.

16. ten Brinke A, Sterk PJ, Masclee AA et al. Risk factors of frequent exacerbations in difficult-to-treat asthma. Eur Respir J. 2005;26:812-8.

17. Kupczyk M, ten Brinke A, Sterk PJ et al. Frequent exacerbators--a distinct phenotype of severe asthma. Clin Exp Allergy. 2014;44:212-21.

18. Green RH, Brightling CE, McKenna S et al. Asthma exacerbations and sputum eosinophil counts: a randomised controlled trial. Lancet. 2002;360:1715-21.

19. Vernon MK, Wiklund I, Bell JA, Dale P, Chapman KR. What do we know about asthma triggers? A review of the literature. J Asthma. 2012;49:991-8.

20. McCarty JC, Ferguson BJ. Identifying asthma triggers. Otolaryngol Clin North Am. 2014;47:109-18.

21. Moore WC, Meyers DA, Wenzel SE et al. Identification of asthma phenotypes using cluster analysis in the Severe Asthma Research Program. Am J Respir Crit Care Med. 2010;181:315-23.

22. Fitzpatrick AM, Teague WG, Meyers DA et al. Heterogeneity of severe asthma in childhood: confirmation by cluster analysis of children in the National Institutes of Health/National Heart, Lung, and Blood Institute Severe Asthma Research Program. J Allergy Clin Immunol. 2011;127:382-9.e1-13.

23. Haldar P, Pavord ID, Shaw DE et al. Cluster analysis and clinical asthma phenotypes. Am J Respir Crit Care Med. 2008;178:218-24.

24. Patrawalla P, Kazeros A, Rogers L et al. Application of the asthma phenotype algorithm from the Severe Asthma Research Program to an urban population. PloS One. 2012;7:e44540.

25. Chapman DG, Irvin CG, Kaminsky DA, Forgione PM, Bates JH, Dixon AE Influence of distinct asthma phenotypes on lung function following weight loss in the obese. Respirology. 2014;19:1170-7.

26. Schatz M, Hsu JW, Zeiger RS et al. Phenotypes determined by cluster analysis in severe or difficult-to-treat asthma. J Allergy Clin Immunol. 2014;133:1549-56. 
27. Howrylak JA, Fuhlbrigge AL, Strunk RC, Zeiger RS, Weiss ST, Raby BA. Classification of childhood asthma phenotypes and long-term clinical responses to inhaled anti-inflammatory medications. J Allergy Clin Immunol. 2014;133:1289-300.

28. Kim TB, Jang AS, Kwon HS et al. Identification of asthma clusters in two independent Korean adult asthma cohorts. Eur Respir J. 2013;41:1308-14.

29. Kaneko Y, Masuko H, Sakamoto T et al. Asthma phenotypes in Japanese adults - their associations with the CCL5 and ADRB2 genotypes. Allergol Int. 2013;62:113-21.

30. Jang AS, Kwon HS, Cho YS et al. Identification of subtypes of refractory asthma in Korean patients by cluster analysis. Lung. 2013;191:87-93.

31. Shaw DE, Sousa AR, Fowler SJ et al. Clinical and inflammatory characteristics of the European U-BIOPRED adult severe asthma cohort. Eur Respir J. 2015;46:1308-21.

32. Fleming L, Murray C, Bansal AT et al. The burden of severe asthma in childhood and adolescence: results from the paediatric U-BIOPRED cohorts. Eur Respir J. 2015;46:1322-33.

33. Lefaudeux D, DMB, Chung KF et al. Clustering analysis of clinical variables in U-BIOPRED adult asthma cohort. Eur Respir J. 2014;44:225.

34. Sutherland ER, Goleva E, King TS et al. Cluster analysis of obesity and asthma phenotypes. PloS One. 2012;7:e36631.

35. Gibeon D, Batuwita K, Osmond M et al. Obesity-associated severe asthma represents a distinct clinical phenotype: analysis of the British Thoracic Society Difficult Asthma Registry Patient cohort according to BMI. Chest. 2013;143:406-14.

36. Amelink M, de Nijs SB, de Groot JC et al. Three phenotypes of adult-onset asthma. Allergy. 2013;68:674-80.

37. Baffi CW, Wood L, Winnica D et al. Metabolic Syndrome and the Lung. Chest. 2016. (Epub ahead of print).

38. Green RH, Brightling CE, Woltmann G, Parker D, Wardlaw AJ, Pavord ID. Analysis of induced sputum in adults with asthma: identification of subgroup with isolated sputum neutrophilia and poor response to inhaled corticosteroids. Thorax. 2002;57(:875-9.

39. Pavord ID, Brightling CE, Woltmann G, Wardlaw AJ. Non-eosinophilic corticosteroid unresponsive asthma. Lancet. 1999;353:2213-4.

40. Jatakanon A, Uasuf C, Maziak W, Lim S, Chung KF, Barnes PJ. Neutrophilic inflammation in severe persistent asthma. Am J Respir Crit Care Med. 1999;160:1532-9.

41. Wenzel SE, Schwartz LB, Langmack EL et al. Evidence that severe asthma can be divided pathologically into two inflammatory subtypes with distinct physiologic and clinical characteristics. Am J Respir Crit Care Med. 1999;160:1001-8.

42. Peters MC, Mekonnen ZK, Yuan S, Bhakta NR, Woodruff PG, Fahy JV. Measures of gene expression in sputum cells can identify TH2-high and TH2-low subtypes of asthma. J Allergy Clin Immunol. 2014;133:388-94.

43. Zhang XY, Simpson JL, Powell $\mathrm{H}$ et al. Full blood count parameters for the detection of asthma inflammatory phenotypes. Clin Exp Allergy. 2014;44:1137-45.

44. Tran TN, Khatry DB, Ke X, Ward CK, Gossage D. High blood eosinophil count is associated with more frequent asthma attacks in asthma patients. Ann Allergy Asthma Immunol. 2014;113:19-24.

45. Ulrik CS, Frederiksen J. Mortality and markers of risk of asthma death among 1,075 outpatients with asthma. Chest. 1995;108:10-5.

46. Malinovschi A, Fonseca JA, Jacinto T, Alving K, Janson C. Exhaled nitric oxide levels and blood eosinophil counts independently associate with wheeze and asthma events in National Health and Nutrition Examination Survey subjects. J Allergy Clin Immunol. 2013;132:821-7.

47. Pavord ID, Korn S, Howarth P et al. Mepolizumab for severe eosinophilic asthma (DREAM): a multicentre, double-blind, placebo-controlled trial. Lancet. 2012;380:651-9.

48. Bel EH, Wenzel SE, Thompson PJ et al. Oral glucocorticoid-sparing effect of mepolizumab in eosinophilic asthma. N Engl J Med. 2014;371:1189-97.

49. Castro M, Mathur S, Hargreave F et al. Reslizumab for poorly controlled, eosinophilic asthma: a randomized, placebo-controlled study. Am J Respir Crit Care Med. 2011;184:1125-32.
50. Castro M, Wenzel SE, Bleecker ER et al. Benralizumab, an anti-interleukin 5 receptor alpha monoclonal antibody, versus placebo for uncontrolled eosinophilic asthma: a phase $2 \mathrm{~b}$ randomised dose-ranging study. Lancet Respir Med. 2014;2:879-90.

51. Hanania NA, Wenzel S, Rosen K et al. Exploring the effects of omalizumab in allergic asthma: an analysis of biomarkers in the EXTRA study. Am J Respir Crit Care Med. 2013;187:804-11.

52. Busse W, Spector S, Rosen K, Wang Y, Alpan O. High eosinophil count: a potential biomarker for assessing successful omalizumab treatment effects. J Allergy Clin Immunol. 2013;132:485-6.

53. Szefler SJ, Phillips BR, Martinez FD et al. Characterization of within-subject responses to fluticasone and montelukast in childhood asthma. J Allergy Clin Immunol. 2005;115:233-42.

54. Kupczyk M, Haque S, Middelveld RJ, Dahlen B, Dahlen SE. Phenotypic predictors of response to oral glucocorticosteroids in severe asthma. Respir Med. 2013;107:1521-30.

55. Dweik RA, Boggs PB, Erzurum SC et al. An official ATS clinical practice guideline: interpretation of exhaled nitric oxide levels (FENO) for clinical applications. Am J Respir Crit Care Med. 2011;184:602-15.

56. Haldar P, Brightling CE, Hargadon B et al. Mepolizumab and exacerbation of refractory eosinophilic asthma. N Engl J Med. 2009;360:973-84.

57. Corren J, Lemanske RF, Hanania NA et al. Lebrikizumab treatment in adults with asthma. N Engl J Med. 2011;365:1088-98.

58. Wenzel S, Ford L, Pearlman D et al. Dupilumab in persistent asthma with elevated eosinophil levels. N Engl J Med. 2013;368:2455-66.

59. Wagener AH, de Nijs SB, Lutter R et al. External validation of blood eosinophils, $\mathrm{FE}(\mathrm{NO})$ and serum periostin as surrogates for sputum eosinophils in asthma. Thorax. 2015;70:115-20.

60. Jia G, Erickson RW, Choy DF et al. Periostin is a systemic biomarker of eosinophilic airway inflammation in asthmatic patients. J Allergy Clin Immunol. 2012;130:647-54.

61. Kanemitsu Y, Matsumoto H, Izuhara K et al. Increased periostin associates with greater airflow limitation in patients receiving inhaled corticosteroids J Allergy Clin Immunol. 2013;132:305-12.

62. Moore WC, Hastie AT, Li X et al. Sputum neutrophil counts are associated with more severe asthma phenotypes using cluster analysis. J Allergy Clin Immunol. 2014;133:1557-63.

63. Moore WC, Bleecker ER, Curran-Everett D et al. Characterization of the severe asthma phenotype by the National Heart, Lung, and Blood Institute's Severe Asthma Research Program. J Allergy Clin Immunol 2007;119:405-13.

64. Wu W, Bleecker E, Moore W et al. Unsupervised phenotyping of Severe Asthma Research Program participants using expanded lung data. J Allergy Clin Immunol. 2014;133:1280-8.

65. Boudier A, Curjuric I, Basagana X et al. Ten-year follow-up of cluster-based asthma phenotypes in adults. A pooled analysis of three cohorts. Am Respir Crit Care Med. 2013;188:550-60.

66. Kupczyk M, Dahlen B, Sterk PJ et al. Stability of phenotypes defined by physiological variables and biomarkers in adults with asthma. Allergy. 2014;69:1198-204.

67. Bourdin A, Molinari N, Vachier I et al. Prognostic value of cluster analysi of severe asthma phenotypes. J Allergy Clin Immunol. 2014;134:1043-50.

68. Woodruff PG, Boushey HA, Dolganov GM et al. Genome-wide profiling identifies epithelial cell genes associated with asthma and with treatment response to corticosteroids. Proc Natl Acad Sci U S A. 2007;104:15858-63.

69. Woodruff PG, Modrek B, Choy DF et al. T-helper type 2-driven inflammation defines major subphenotypes of asthma. Am J Respir Crit Care Med. 2009;180:388-95.

70. Baines KJ, Simpson JL, Wood LG et al. Sputum gene expression signature of 6 biomarkers discriminates asthma inflammatory phenotypes. J Allergy Clin Immunol. 2014;133:997-1007.

71. Modena BD, Tedrow JR, Milosevic J et al. Gene expression in relation to exhaled nitric oxide identifies novel asthma phenotypes with unique biomolecular pathways. Am J Respir Crit Care Med. 2014;190:1363-72. 
72. Kuo C-HS, PS, Loza M et al. Asthma Phenotypes From Semi-Supervised Machine-Learning Approach Of Bronchial Biopsy And Brush Transcriptomics In U-BIOPRED. Am J Respir Crit Care Med. 191:A2205.

73. Zhang Q, Illing R, Hui CK et al. Bacteria in sputum of stable severe asthma and increased airway wall thickness. Respir Res. 2012;13:35.

74. Wood LG, Simpson JL, Hansbro PM, Gibson PG. Potentially pathogenic bacteria cultured from the sputum of stable asthmatics are associated with increased 8-isoprostane and airway neutrophilia. Free Radic Res. 2010; 44:146-54.

75. Fitzpatrick AM, Holguin F, Teague WG, Brown LA. Alveolar macrophage phagocytosis is impaired in children with poorly controlled asthma. J Allergy Clin Immunol. 2008;121:1372-8.

76. Liang Z, Zhang $\mathrm{Q}$, Thomas $\mathrm{CM}$ et al. Impaired macrophage phagocytosis of bacteria in severe asthma. Respir Res. 2014;15:72.

77. Goleva E, Jackson LP, Harris JK et al. The effects of airway microbiome on corticosteroid responsiveness in asthma. Am J Respir Crit Care Med. 2013;188:1193-201.

78. Nguyen LT, Lim S, Oates T, Chung KF. Increase in airway neutrophils after oral but not inhaled corticosteroid therapy in mild asthma. Respir Med. 2005;99:200-7.

79. Shannon J, Ernst P, Yamauchi Y et al. Differences in airway cytokine profile in severe asthma compared to moderate asthma. Chest. 2008;133:420-6.

80. Al-Ramli W, Prefontaine D, Chouiali F et al. T(H)17-associated cytokines (IL-17A and IL-17F) in severe asthma. J Allergy Clin Immunol. 2009;123:1185-7.

81. McKinley L, Alcorn JF, Peterson A et al. TH17 cells mediate steroid-resistant airway inflammation and airway hyperresponsiveness in mice. J Immunol. 2008;181:4089-97.

82. Busse WW, Holgate S, Kerwin E et al. Randomized, Double-Blind, Placebo-controlled Study of Brodalumab, a Human Anti-IL-17 Receptor
Monoclonal Antibody, in Moderate to Severe Asthma. Am J Respir Crit Care Med. 2013;188:1294-302.

83. Simpson JL, Phipps S, Baines KJ, Oreo KM, Gunawardhana L, Gibson PG. Elevated expression of the NLRP3 inflammasome in neutrophilic asthma. Eur Respir J. 2014;43:1067-76.

84. Pavlidis S LM, Baribaud F, Kuo C-HS et al. Sputum Transcriptomics Provides Inflammasome And Th2-high Gene Signatures Associated With Eosinophilia In The U-BIOPRED Adult Severe Asthma Cohort. Am J Respir Crit Care Med. 2015;191:A3623.

85. Chung KF. Targeting the interleukin pathway in the treatment of asthma Lancet. 2015;386:1086-96.

86. Gibeon D, Chung KF. The investigation of severe asthma to define phenotypes. Clin Exp Allergy. 2012;42:678-92.

87. Flood-Page P, Swenson C, Faiferman I et al. A study to evaluate safety and efficacy of mepolizumab in patients with moderate persistent asthma. Am J Respir Crit Care Med. 2007;176:1062-71.

88. Nair P. Anti-interleukin-5 monoclonal antibody to treat severe eosinophilic asthma. N Engl J Med. 2014;371:1249-51.

89. Castro M, Zangrilli J, Wechsler ME et al. Reslizumab for inadequately controlled asthma with elevated blood eosinophil counts: results from two multicentre, parallel, double-blind, randomised, placebo-controlled, phase 3 trials. Lancet Respir Med. 2015;3:355-66.

90. Chung KF. New treatments for severe treatment-resistant asthma: targeting the right patient. Lancet Respir Med. 2013;1:639-52.

91. De Meulder B, Lefaudeux D BJ, Loza MJ et al. The first U-BIOPRED blood handprint of severe asthma. Eur Respir J. 2015;46:PA4889.

92. Fu JJ, McDonald VM, Baines KJ, Gibson PG. Airway IL-1 beta and systemic inflammation as predictors of future exacerbation risk in asthma and COPD. Chest. 2015;148;618-29. 